Pacific Journal of Mathematics

ON MALCEV ALGEBRAS 


\title{
ON MALCEV ALGEBRAS
}

\author{
T. S. RAVISANKAR
}

A Malcev algebra is an anticommutative algebra satisfying the identity $(x y)(x z)=((x y) z) x+((y z) x) x+((z x) x) y$. Various notions of solvability have been introduced for a Malcev algebra mainly with the aim of proving the following result or one of its corollaries for the related notion of semisimplicity.

THEOREM A. A semisimple Malcev algebra over a field of characteristic zero is a direct sum of ideals which are simple as algebras.

In the present paper all these notions of solvability are shown to be equivalent. This fact enables simplifications of proofs of some known results. The Killing form of a Malcev algebra is considered and is shown to possess some of the nice properties of the classical Cartan-Killing form of a Lie algebra. Finally, the structure of the Malcev algebra is studied in relation to that of the derivation algebra of an associated Lie triple system.

The equivalence of the notions of solvability is proved in $\S 1$ of the paper (Theorem 1.1). This result is used in $\S 2$ to give an alternative proof of Theorem A. This equivalence is further used to simplify the proofs of certain results of Loos [6] and to throw some light on a conjecture of Sagle [7]. In $\S \S 3,4$ we consider the Killing form of a Malcev algebra over a field of characteristic prime to six. The equivalence (Theorem 3.1) of the nondegeneracy of the Killing forms of a Malcev algebra and of the (standard) enveloping Lie algebra of its associated Lie triple system enables us to extend to Malcev algebras an important result of Zassenhaus for Lie algebras. Other results in these sections are in the nature of extensions or analogues for Malcev algebras of known results for Lie algebras. In $\$ 5$ we consider the derivation algebra of the Lie triple system associated to a Malcev algebra over a field of characteristic zero. In the absence of any result connecting the semisimplicity of the derivation algebra to that of the Malcev algebra itself, Theorem 5.1 of this section seems to be the only one of its class.

1. Equivalence of the variants of the notion of solvability. Let $A$ be a finite dimensional Malcev algebra (as is assumed throughout the present paper) over a field $F$ of characteristic prime to six. Let $T_{A}$ be the Lie triple system associated to $A$ by defining the composition $[x, y, z]=2(x y) z-(y z) x-(z x) y$ in the vector space $A$ (see [6]); let $T^{\prime}$ be the general Lie triple system (see [8]) formed by the space $A$ 
with respect to the compositions $x y$ and $[x, y, z]_{1}=(x y) z-(y z) x-$ $(z x) y$. For $x, y, z$ in $A$, we denote by $J(x, y, z)$, the element $(x y) z+$ $(y z) x+(z x) y$. Then if $B$ is an ideal of $A$, so are $B B+(B B) A,\left[T_{A}\right.$, $B, B],[B, B, B], B B+\left[T^{\prime}, B, B\right]_{1}$ and $B B+J(B, B, A)$. The notions of solvability arising from the iteration of these ideals are respectively called $L$-solvability (see [4]), solvability as an ideal of the Lie triple system $T_{A}$, weak (schwach) solvability as an ideal of $T_{A}$ (see [6]), $Y$-solvability (in the sense of Yamaguti [8]) and strong solvability. Then we have the

THEOREM 1.1. For an ideal $B$ of $A$, the following statements are equivalent.
(i) $B$ is solvable.
(ii) $B$ is $L$-solvable.
(iii) $B$ is solvable as an ideal of $T_{A}$.
(iv) $B$ is weak solvable as an ideal of $T_{A}$.
(v) $B$ is $Y$-solvable.
(vi) $B$ is strongly solvable.

Proof. (i) $\Leftrightarrow$ (ii) is essentially a recently proved result ([4], Lemma 2). Further, for an ideal $B$ of $A$ we have

$$
B B \cong B B+\left[T^{\prime}, B, B\right]_{1}=B B+J(B, B, A)=B B+(B B) A .
$$

The iteration of these inclusions immediately yields the equivalence of (i), (ii), (v) and (vi), from the equivalence of (i) and (ii). That (iii) $\Rightarrow$ (i) is known. (See the first part of the proof of [6], Satz 2; we note that this part of the proof holds even for the characteristic of $F \neq 2,3$, though not explicitly stated there.) Again, for the ideal $B$

$$
\left[T_{A}, B, B\right] \subseteq 2\left(T_{A} B\right) B+(B B) T_{A}+\left(B T_{A}\right) B \subseteq B B+(B B) A .
$$

By iteration, this leads to (i) $\Rightarrow$ (iii) in view of the equivalence of (i) and (ii). Again (iv) $\Rightarrow$ (i) is a known implication (see [6], Korollar 5). It is further evident that (i) $\Rightarrow$ (iii) $\Rightarrow$ (iv). The theorem is completely proved.

REMARK 1. In Theorem 1.1, (i) $\Rightarrow$ (iii) is a considerable improvement of the same implication proved in [6], when $B$ is an ideal of a Malcev algebra $A$ over a field of characteristic zero. This restriction on the characteristic of the base field, involved heavily in the proof in [6], is superfiuous for arriving at this implication, as well as (iv) $\Rightarrow$ (i).

2. At this point we refer the reader to the notions of $J$-potency and $J_{1}$-potency introduced by Sagle (see [7], §6). For the sake of convenience we make a departure from Sagle's notions by omitting the additional assumption that the index of $J_{i}$-potency is greater than 
or equal to 2. This omission amounts to defining any Lie ideal of a Malcev algebra to be $J$-potent. If $B$ is a solvable ideal of $A$, and consequently $L$-solvable by Theorem 1.1 , then $B$ is $J_{1}$-potent. For, $J_{1}(B)=J(B, B, A) \subseteq B B+(B B) A=B^{(1)}$; by induction $J_{1}^{k+1}(B)=J\left(J_{1}^{k}(B)\right.$, $\left.J_{1}^{k}(B), A\right) \subseteq B^{(k)} B^{(k)}+\left(B^{(k)} B^{(k)}\right) A=B^{(k+1)}$. In particular, the maximal solvable ideal of $A$, or its radical $R$, is contained in the maximal $J_{1}$-potent ideal $\widetilde{\Im}_{1}(A)$ of $A$. The preceding argument with $J_{1}$-potency replaced by $J$-potency $(\widetilde{S}(A)$ being the $J$-radical) along with Theorem $A$, leads to the formal converse of a result of Sagle ([7], Theorem 6.6): For a Malcev algebra $A$ over a field of characteristic zero, $\Im(A)=0$ implies that $A$ is non-Lie, and that $A$ is a direct sum of simple ideals. On the other hand, if we retain Sagle's definition of the concept of $J$ potency, we are able to assert his conjecture (see [7], §1) that the actual converse of his Theorem 6.6 does not hold. For this assertion we need only consider the example of an algebra composed of the direct sum (as ideals) of a simple non-Lie Malcev algebra and a nonsemisimple Lie algebra.

2. Simple proof of Theorem A. The use of Theorem 1.1 in the proof of Theorem A is actually in Lemma 2.1, which in turn leads to Proposition 2.2.

We define the $C$-radical of a Malcev algebra (Lie triple system) to be its maximal solvable characteristic ideal and observe that the $C$-radical is contained in the radical. Further, any ideal of a Malcev algebra $A$ is an ideal of the Lie triple system $T_{A}$; any characteristic ideal of $T_{A}$ is a characteristic ideal of $A$ (see [6], Lemma 2 and Bemerkung following it). Thus we have, in view of Theorem 1.1, the following

Lemma 2.1. $C$-radical of $T_{A} \subseteq C$-radical of $A \subseteq$ radical of $A \subseteq$ radical of $T_{A}$.

In case the base field is of zero characteristic, the radical of $T_{A}$ is a characteristic ideal ([6], Lemma 5). We can then replace the inclusions in Lemma 2.1 by equalities. This observation immediately leads to

Proposition 2.2 (cf. [6], Satz 2). Over a field of characteristic zero, $A$ is semisimple iff $T_{A}$ is semisimple; more explicitly, the radicals of $A$ and $T_{A}$ coincide.

Proof of Theorem A. We first observe that if $T_{A}$ is simple for a Malcev algebra $A$, then $A$ is itself simple. (Any ideal of $A$ is an ideal of $T_{A}$ and $A A \neq 0$.) Theorem A now follows immediately from 
this observation, Proposition 2.2 and the analogue of Theorem A itself for Lie triple systems ([5], Theorem 2.9).

We have the following corollaries.

COROLlaRy 2.3 (cf. [6], Korollar 2). Over a field of characteristic zero, $A$ is simple iff $T_{A}$ is simple.

Corollary 2.4 (cf. [6], Korollar 3 and [8], Theorem 2.3). Every derivation of a semisimple Malcev algebra $A$ over a field of characteristic zero is inner.

Proof. $T_{A}$ is semisimple, by Proposition 2.2. Hence, every derivation of $T_{A}$ is inner ([5], Theorem 2.11). Let $L_{x}$ or $L(x)$ denote the left multiplication in $A$ and let $D$ be a derivation of $A$. Then $D$ is a derivation of $T_{A}$ so that $D=\sum_{i=1}^{n} R\left(x_{i}, y_{i}\right)$ for some $x_{i}, y_{i}$ in $A$. $\left(R(x, y)\right.$ is the mapping $z \rightarrow[x, y, z]=z\left(2 L_{x y}-\left[L_{x}, L_{y}\right]\right)$ of $A$ into A.) Since the Lie multiplication algebra $\mathscr{L}(A)$ of $A$ is $L(A)+[L(A)$, $L(A)$ ] ([7], Theorem 5.1), we have $D \in \mathscr{L}(A)$; or that $D$ is an inner derivation of $A$.

3. The Killing form of a Malcev algebra. Let $A$ be a Malcev algebra over a field of characteristic prime to six and $T_{A}$ be the Lie triple system associated with it. Let $L=T_{A} \oplus\left[T_{A}, T_{A}\right]$ be the enveloping Lie algebra of $T_{A}$ obtained by the standard construction (or imbedding) (see [2], [5]). This construction can be essentially described as follows: $L=T_{A} \oplus D_{0}\left(T_{A}\right)$ (vector space direct sum), where $D_{0}\left(T_{A}\right)$ is the Lie algebra of all inner derivations of $T_{A}$, i.e. derivations of the form $\sum R\left(x_{i}, y_{i}\right) ; R(x, y): z \rightarrow[x, y, z]$. The composition in $L$ is defined by (see [6])

$$
\begin{aligned}
& {[x, y]=R(x, y)} \\
& {[D, x]=-[x, D]=x D} \\
& {\left[D_{1}, D_{2}\right]=D_{1} D_{2}-D_{2} D_{1}}
\end{aligned}
$$

for $x, y$ in $T_{A}$ and $D, D_{1}, D_{2}$ in $D_{0}\left(T_{A}\right)$. Actually $T_{A}$ is the subspace of skewsymmetric elements, and $\left[T_{A}, T_{A}\right] \equiv D_{0}\left(T_{A}\right)$ the subspace of symmetric elements with respect to an uniquely defined involutory automorphism $\sigma$ in $L$. Let $\beta(u, v)=$ trace ad $u$ ad $v$ be the Killingform of $L$ and $\alpha(x, y)=$ trace $L_{x} L_{y}\left(L_{x}\right.$ stands for the left multiplication in $A$.) the Killing form of $A$. As is already known (see [6], Lemma 6), $6 \alpha(x, y)=\beta(x, y)$ for $x, y$ in $A$. Further, we observe that the spaces $T_{A}$ and $\left[T_{A}, T_{A}\right]$ are orthogonal to each other relative to the Killing form $\beta$ of $L$. We now prove the 
Theorem 3.1. If $A$ is a Malcev algebra over a field of characteristic prime to six, then the Killing form of $A$ is nondegenerate iff the Killing form of $L$ is nondegenerate.

Proof. Firstly, suppose that the Killing form $\beta$ of $L$ is nondegenerate. If possible, let $x \in A$ and $\alpha(x, y)=0$ for all $y$ in $A$. Then $\beta(x, y)=0$ for all $y$ in $A$; but $\beta\left(x,\left[T_{A}, T_{A}\right]\right)=0$ so that $\beta(x, L)=0$. $\beta$ being nondegenerate, $x=0$. Thus the Killing form $\alpha$ of $A$ is also nondegenerate.

Now, to prove the reverse implication, let the Killing form $\alpha$ of $A$ be nondegenerate. Let $z+\sum\left[x_{i}, y_{i}\right]$ be an element of $L \equiv T_{A} \oplus$ $\left[T_{A}, T_{A}\right]$ such that $\beta\left(z+\sum\left[x_{i}, y_{i}\right], L\right)=0$. In particular, $\beta\left(z+\sum\left[x_{i}\right.\right.$, $\left.\left.y_{i}\right], T_{A}\right)=0$, i.e. $\beta\left(z, T_{A}\right)=0$. But $z \in T_{A}$, so that $\alpha\left(z, T_{A}\right)=0$, since the characteristic of the base field is prime to six. Since the Killing form of $A$ is nondegenerate this is possible only if $z=0$. Hence we have $\beta\left(\sum\left[x_{i}, y_{i}\right], L\right)=0$. If, for instance, $u, v$ are arbitrary elements in $T_{A}, \beta\left(\sum\left[x_{i}, y_{i}\right],[u, v]\right)=0$. Since the Killing form of a Lie algebra is associative ([3], p. 71), we have that $\beta\left(\left[\sum\left[x_{i}, y_{i}\right], u\right], v\right)=0$. But $\left[\sum\left[x_{i}, y_{i}\right], u\right]$ belongs to $T_{A}$, and so $\alpha\left(\left[\sum\left[x_{i}, y_{i}\right], u\right], v\right)=0$. This coupled with the assumption that the Killing form of $A$ is nondegenerate, yields $\left[\sum\left[x_{i}, y_{i}\right], u\right]=0$. Since $u$ is arbitrary in $T_{A}$, it now follows from the definition of standard imbedding (see [2], [5] and beginning of this section) that $\sum\left[x_{i}, y_{i}\right]=0$. In other words, the Killing form of $L$ is nondegenerate and the proof of the theorem is complete.

REMARK 1. The standard construction is used only in the "only if" part of the above theorem. The "if" part just requires that $T_{A}$ be imbedded as the subspace of skewsymmetric elements with respect to an involutory automorphism in a Lie algebra $L$.

CoRollary 3.2 (cf. [6], Theorem A). The Killing form of a semisimple Malcev algebra $A$ over a field of characteristic zero is nondegenerate.

Proof. $T_{A}$ is a semisimple Lie triple system by Proposition 2.2. Consequently the (standard) enveloping Lie algebra $L$ is semisimple (see [5], Theorem 2.7). Further, the Killing form of $L$ is nondegenerate ([3], pp. 69, 70). Now, the "if" part of the above Theorem 3.1 immediately yields the corollary.

REMARK 2. Theorem A can be also deduced from Corollary 3.2, using Dieudonné's theorem ([3], p. 71, Theorem 3.3; see also [7], Theorem 7.20). Note that Corollary 3.2 incidentally leads to the characterization (see [6]): A simple non-Lie Malcev algebra over an 
algebraically closed field of characteristic zero is isomorphic to the 7-dimensional Malcev algebra constructed by Sagle ([7], Example 3.2).

REMARK 3. If $R$ is the radical of a Malcev algebra $A$ over a field of characteristic zero, then $R$ is also the radical of $T_{A}$, by Proposition 2.2. We can then use the characterization of the radical of the (standard) enveloping Lie algebra $L$ due to Cartan-Harish-Chandra ([3], p. 73, Theorem 3.5), to obtain the following analogue of the same theorem for Malcev algebras: $R \equiv\left\{x \in A \mid \alpha\left(x,\left[T_{A}, T_{A}, T_{A}\right]\right)=0\right\}$ (see [6], p. 561, 1. 7). We note that this characterization of the radical itself would suffice to deduce Corollary 3.2 (or [6], Theorem A) directly.

The following analogue for Malcev algebras of a known criterion for solvability for a Lie algebra (see [3], p. 69) is a trivial consequence of the above characterization of the radical and Theorem 1.1.

Proposition 3.3. A Malcev algebra $A$ over a field of characteristic zero is solvable iff $\alpha(x, x)=0$ for all $x$ in $\left[T_{A}, T_{A}, T_{A}\right]$.

REMARK 4. Satz 3 of [6] enables us to replace $\left[T_{A}, T_{A}, T_{A}\right]$ in Proposition 3.3 by $A^{(k)}$ for $k \geqq 3$, where $A^{(1)}=A, A^{(2)}=A^{(1)} A^{(1)}, \cdots$, $A^{(k+1)}=A^{(k)} A^{(k)}, \cdots$ is the derived series of $A$.

4. Extension of a theorem of Zassenhaus. As an application of Theorem 3.1, we shall give in this section an extension, to Malcev algebras, of a theorem of Zassenhaus for Lie algebras ([3], p. 74, Theorem 3.6).

Let $A$ be a Malcev algebra over a field of characteristic prime to six such that the Killing form $\alpha$ of $A$ is nondegenerate. Then, the Killing form $\beta$ of the enveloping Lie algebra $L$ of $T_{A}$ (standard imbedding) is nondegenerate by Theorem 3.1. Hence, every derivation of $L$ is inner, by a known result ([3], Theorem 3.6). Now, suppose $D$ is a derivation of $T_{A}$. Define a mapping $D^{\prime}$ on $\left[T_{A}, T_{A}\right]$ by specifying $([x, y]) D^{\prime}=[x D, y]+[x, y D]$ for $x, y$ in $T_{A}$, and extending linearly to $\left[T_{A}, T_{A}\right]$. Then, the mapping $D^{\prime}$ is well-defined (see [5]). For, if $\sum\left[x_{i}, y_{i}\right]=\sum\left[x_{j}^{\prime}, y_{j}^{\prime}\right]$ for $x_{i}, y_{i}, x_{j}^{\prime}, y_{j}^{\prime}$ in $T_{A}$, then $\left[\sum\left[x_{i}, y_{i}\right]-\sum\left[x_{j}^{\prime}\right.\right.$, $\left.\left.y_{j}^{\prime}\right], t\right]=0$ for all $t$ in $T_{A}$, i.e. $\sum\left[x_{i}, y_{i}, t\right]-\sum\left[x_{j}^{\prime}, y_{j}^{\prime}, t\right]=0$. Since $D$ is a derivation of $T_{A}$, we have $\sum\left[x_{i} D, y_{i}, t\right]+\sum\left[x_{i}, y_{i} D, t\right]-\sum\left[x_{j}^{\prime} D\right.$, $\left.y_{j}^{\prime}, t\right]-\sum\left[x_{j}^{\prime}, y_{j}^{\prime} D, t\right]+\sum\left[x_{i}, y_{i}, t D\right]-\sum\left[x_{j}^{\prime}, y_{j}^{\prime}, t D\right]=0$; hence $\left[\left(\sum\left[x_{i}\right.\right.\right.$, $\left.\left.\left.y_{i}\right]-\sum\left[x_{j}^{\prime}, y_{j}^{\prime}\right]\right) D^{\prime}, t\right]=0$. By the definition of standard imbedding (see [2], [5] and the beginning of $\S 3$ ), we have $\left(\sum\left[x_{i}, y_{i}\right]\right) D^{\prime}=\left(\sum\left[x_{j}^{\prime}\right.\right.$, $\left.\left.y_{j}^{\prime}\right]\right) D^{\prime}$; in other words $D^{\prime}$ is single-valued. The mapping $D^{\prime}$ determines a derivation $\bar{D}$ on $L$ extending $D$ so that $\bar{D}=\operatorname{ad} a$ for some $a$ in $L$. (ad $a$ is the mapping $b \rightarrow[a, b]$ of $L$ into itself.) Now, since $\bar{D}$ 
restricted to $T_{A}$ maps $T_{A}$ into itself, for $x$ in $T_{A},[a, x]$ belongs to $T_{A}$, i.e. $a$ should belong to $\left[T_{A}, T_{A}\right]$. This means precisely that $D$ is an inner derivation of $T_{A}$. This observation leads as in the proof of Corollary 2.4 to the following extension of the theorem of Zassenhaus.

THEOREM 4.1. If the Killing form of a Malcev algebra A over a field of characteristic prime to six is nondegenerate, then every derivation of $A$ is inner.

Remark. Since many of the identities for a Malcev algebra are known only with one or other of the restrictions of characteristic $\neq$ 2,3 on the base field, it does not seem likely that one can dispense with these restrictions in Theorem 4.1.

5. The derivation algebra $D\left(T_{A}\right)$. In this section we consider the derivation algebra $D\left(T_{A}\right)$ of the Lie triple system $T_{A}$ associated with a Malcev algebra $A$ over a field of characteristic zero.

TheORem 5.1. A Malcev algebra $A$ over a field $F$ of characteristic zero is semisimple iff $D\left(T_{A}\right)$ is a semisimple Lie algebra.

Proof. Firstly let $A$ be semisimple. Then, by Proposition 2.2, $T_{A}$ is a semisimple Lie triple system. Hence, every derivation of $T_{A}$ is inner (see [5], Theorem 2.11). By [6], Lemma 2, $D\left(T_{A}\right)=\mathscr{L}(A)$, the Lie multiplication algebra of $A$ (see also [6], p. 558). But $\mathscr{L}(A)$ is known to be semisimple ([7], Corollary 5.32); $D\left(T_{A}\right)$ is semisimple. This proves the "only if" part of the theorem.

Conversely, let $D\left(T_{A}\right)$ be semisimple, for a Malcev algebra $A$ over the field $F$. Then $D\left(T_{A}\right)$ is completely reducible in $T_{A}$ (see [3], p. 81, Theorem 3.10), so that $T_{A}$ is a direct sum of $D\left(T_{A}\right)$-irreducible subspaces $T_{i}$ of $T_{A}$. $\quad T_{i}$ are then characteristic ideals of $T_{A}$ (see [6], Bemerkung following Lemma 2), such that each $T_{i}$ has no characteristic ideals other than $T_{i}$ and 0 . Now $\left[T_{i}, T_{i}, T_{i}\right]$ is a characteristic ideal of $T_{i}$ which should therefore be either 0 or $T_{i}$. In the latter case, the radical $R_{i}$ of $T_{i}$ over the field $F$ of characteristic zero is a characteristic ideal ([6], Lemma 5); since $\left[T_{i}, T_{i}, T_{i}\right]=T_{i}, T_{i}$ is not solvable so that $R_{i}=0$ and $T_{i}$ is semisimple. In this case every derivation of $T_{i}$ is inner ([5], Theorem 2.11), so that any ideal of $T_{i}$ is a characteristic ideal of $T_{i}$. In other words, $T_{i}$ is simple in this case. Thus we have $T_{A}=C \oplus T^{\prime}$, where $C \equiv\left\{x \in T_{A} \mid\left[x, T_{A}, T_{A}\right]=0\right\}$ and $T^{\prime}$ is a direct sum of simple ideals. If $C \neq 0$, then the mapping $I: y+t \rightarrow y \equiv$ $(y+t) I$ (for $y \in C, t \in T^{\prime}$ ) of $T_{A}$ into itself is a derivation of $T_{A}$; the subspace $\{\alpha I\}_{\alpha \in F}$ is a solvable (in fact, central) ideal of $D\left(T_{A}\right)$, a

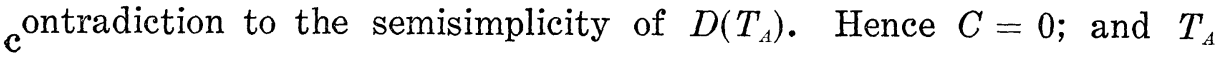


is semisimple. $A$ is therefore semisimple (by Proposition 2.2).

REMARK 1. The above theorem is somewhat analogous to a result of Hochschild ${ }^{1}$ ([1]): An associative (Lie) algebra over a field $F$ of characteristic zero is semisimple iff its derivation algebra is semisimple. Incidentally we note that, for the case of Lie algebras, this result can be proved as follows. If $L$ is a semisimple Lie algebra, $x \rightarrow \operatorname{ad} x$ is a faithful mapping and $D(L)=\{\operatorname{ad} x\}_{x \in L}$ is semisimple. Conversely, if $D(L)$ is semisimple for a Lie algebra $L$, $\{\operatorname{ad} x\}_{x \in L}$, being an ideal of $D(L)$, is semisimple. Hence $\{\operatorname{ad} L\}$ is completely reducible in $L$ ([3], Theorem 3.10); $L$ is thus reductive, $L=$ center $C^{\prime} \oplus$ the semisimple ideal $[L, L] . \quad$ As in the second part of the proof of Theorem 5.1, if $C^{\prime} \neq 0$, the linear mapping which coincides with the identity map on $C^{\prime}$ and 0 on $[L, L]$ is a derivation $D$ of $L$; then $\{\alpha D\}_{\alpha \in F}$ is a solvable (central) ideal of $D(L)$ giving rise to a contradiction, thus proving that $L$ is semisimple.

REMARK 2. As an illustration of Theorem 5.1, we note that the derivation algebra $D\left(T_{A}\right)$ associated with the 7-dimensional Malcev algebra $A$ constructed by Sagle [7], is a 21-dimensional simple Lie algebra of type $B_{3}$. In fact, $D\left(T_{A}\right)=\mathscr{L}(A)$ [6, Lemma 2] $=\Delta(A, A)$ (in the notation of Sagle [7], Theorem 5.9) is a simple Lie algebra of dimension $21\left[7\right.$, p. 455]. That it is of type $B_{3}$ follows from [3, Theorem 4.7], using the nondegeneracy of the Killing form of $A$.

\section{REFERENCES}

1. G. Hochschild, Semisimple algebras and generalized derivations, Amer. J. Math., 64 (1942), 677-694.

2. N. Jacobson, General representation theory for Jordan algebras, Trans. Amer. Math. Soc., 70 (1951), 509-530.

3. - Lie Algebras, Interscience, New York, 1962.

4. E. N. Kuz'min, The locally nilpotent radical of a Malcev algebra satisfying the $n^{\text {th }}$ Engel condition, Soviet Math. Dokl. 8 (1967), 1434-1436.

5. W. G. Lister, A structure theory of Lie triple systems, Trans. Amer. Math. Soc., 72 (1952), 217-242.

6. O. Loos, Über eine Beziehung zwischen Malcev-algebren und Lie-Tripelsystemen, Pacific J. Math., 18 (1966), 553-562.

7. A. A. Sagle, Malcev algebras, Trans. Amer. Math. Soc., 101 (1961), 426-458.

8. K. Yamaguti, On the theory of Malcev algebras, Kumamoto J. Sci., A 6, (1963-65), $9-45$.

Received September 19, 1969 and in revised form March 23, 1972.

Birla Institute of TeChnology and Science

PILANI, INDIA

1 The author's familiarity with Hochschild's paper is only through the Mathematical Reviews Vol. 4 (1943), 71-72. 


\section{PACIFIC JOURNAL OF MATHEMATICS}

\section{EDITORS}

H. SAMELSON

Stanford University

Stanford, California 94305

C. R. HobBY

University of Washington

Seattle, Washington 98105
J. DUGUNDJI

Department of Mathematics

University of Southern California

Los Angeles, California 90007

RICHARD ARENS

University of California

Los Angeles, California 90024

\section{ASSOCIATE EDITORS}

E. F. BECKENBACH

B. H. NeumanN

F WoLF

K. YoshidA

\section{SUPPORTING INSTITUTIONS}

UNIVERSITY OF BRITISH COLUMBIA

UNIVERSITY OF SOUTHERN CALIFORNIA

CALIFORNIA INSTITUTE OF TECHNOLOGY

UNIVERSITY OF CALIFORNIA

MONTANA STATE UNIVERSITY

STANFORD UNIVERSITY

UNIVERSITY OF NEVADA

NEW MEXICO STATE UNIVERSITY

OREGON STATE UNIVERSITY

UNIVERSITY OF OREGON

OSAKA UNIVERSITY

UNIVERSITY OF TOKYO

UNIVERSITY OF UTAH

WASHINGTON STATE UNIVERSITY

UNIVERSITY OF WASHINGTON

$\stackrel{*}{*} \stackrel{*}{*} \stackrel{*}{*}{ }^{*}{ }^{*}$ MMEICAN MATHEMATICAL SOCIETY

NAVAL WEAPONS CENTER

Printed in Japan by International Academic Printing Co., Ltd., Tokyo, Japan 


\section{Pacific Journal of Mathematics}

\section{Vol. 42, No. $1 \quad$ January, 1972}

Tage Bai Andersen, On Banach space valued extensions from split faces ........

David Marion Arnold, A duality for quotient divisible abelian groups of finite

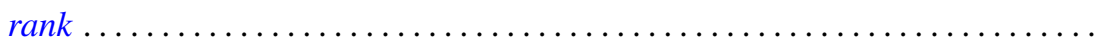

Donald Pollard Ballou, Shock sets for first order nonlinear hyperbolic

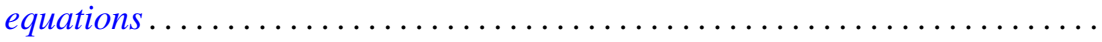

Leon Brown and Lowell J. Hansen, On the range sets of $H^{p}$ functions .........

Alexander Munro Davie and Arne Stray, Interpolation sets for analytic

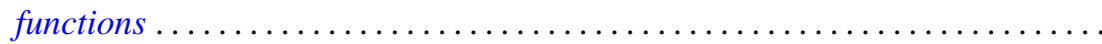

M. G. Deshpande, Structure of right subdirectly irreducible rings. II . . . . . . . . .

Barry J. Gardner, Some closure properties for torsion classes of abelian

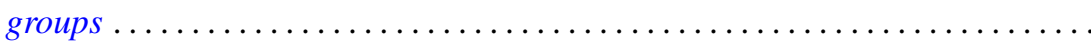

Paul Daniel Hill, Primary groups whose subgroups of smaller cardinality are

direct sums of cyclic groups . . . . . . . . . . . . . . . . . . .

Richard Allan Holzsager, When certain natural maps are equivalences .........

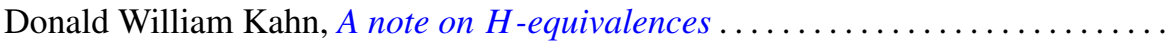

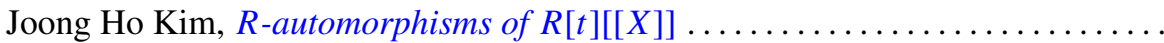

Shin'ichi Kinoshita, On elementary ideals of polyhedra in the 3-sphere.........

Andrew T. Kitchen, Watts cohomology and separability...

Vadim Komkov, A technique for the detection of oscillation of second order

ordinary differential equations .

Charles Philip Lanski and Susan Montgomery, Lie structure of prime rings of characteristic 2

Andrew Lenard, Some remarks on large Toeplitz determinants . .

Kathleen B. Levitz, A characterization of general Z.P.I.-rings. II .

Donald A. Lutz, On the reduction of rank of linear differential systems

David G. Mead, Determinantal ideals, identities, and the Wronskian ...

Arunava Mukherjea, A remark on Tonelli's theorem on integration in product

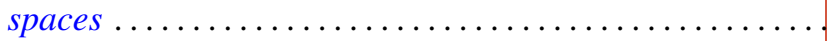

Hyo Chul Myung, A generalization of the prime radical in nonassociative rings.

John Piepenbrink, Rellich densities and an application to unconditionally nonoscillatory elliptic equations.

Michael J. Powers, Lefschetz fixed point theorems for a new class of multi-valued maps .

Aribindi Satyanarayan Rao, On the absolute matrix summability of a Fourier

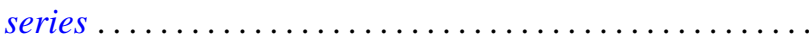

T. S. Ravisankar, On Malcev algebras ......................... 227

William Henry Ruckle, Topologies on sequences spaces . . . . . . . . . . . . . 235

Robert C. Shock, Polynomial rings over finite dimensional rings . . . . . . . . . 251

Richard Tangeman, Strong heredity in radical classes . . . . . . . . . . . . . . 259

B. R. Wenner, Finite-dimensional properties of infinite-dimensional spaces . . . . 267 